\title{
A Fuzzy GA Based STATCOM for Power Quality Improvement
}

\author{
S. Deepa, K. P. Nithya, C. S. Ajin Sekar, V. Deepalakshmi \\ Department of Electrical and Electronics Engineering, Panimalar Institute of Technology, India
}

\begin{tabular}{l} 
Article Info \\
\hline Article history: \\
Received Jun 8, 2017 \\
Revised Aug 19, 2017 \\
Accepted Aug 24, \\
\hline Keyword: \\
FLC \\
Genetic algorithm \\
STATCOM \\
THD \\
Voltage Sag
\end{tabular}

Article Info

Article history:

Received Jun 8, 2017

Revised Aug 19, 2017

Accepted Aug 24, 2017

\section{Genetic algorithm \\ STATCOM \\ Voltage Sag}

\begin{abstract}
This paper deals with various power issues such as voltage sag, swell, harmonics, and surges using static synchronous compensator (STATCOM). The conventional controller suffers from uncertain parameters and non-linear qualities. However they are computationally inefficient extending to optimize the fuzzy controller (FC) parameters, since they exhaustively search the optimal values to optimize the objective functions. To overcome this drawback, a genetic algorithm (GA) based Fuzzy controller parameter optimization is presented in this paper. The GA algorithm is used to find the optimal fuzzy parameters for minimizing the objective functions. The feasibility of the proposed GA technique for distribution systems to improve the sag and total harmonic Distortion (THD) as major power quality indices in sensitive loads at fault conditions has been simulated and tested. Therefore, the multi-objective optimization algorithm is considered in order to attain a better performance in solving the related problems.
\end{abstract}

Copyright $@ 2017$ Institute of Advanced Engineering and Science. All rights reserved.

\section{Corresponding Author:}

S. Deepa,

Department of Electrical and Electronics Engineering,

Panimalar Institute of Technology,

Bangalore Trunk Road, Ponnamalle, Chennai, Tamilnadu, India.

Email: dee_soms123@yahoo.co.in

\section{INTRODUCTION}

Power quality problems in industrial applications concern a wide range of disturbances such as voltage sags and swells, flicker, interruptions, harmonic distortion. Preventing such phenomena is particularly important because of the increasing heavy automation in almost all the industrial processes. Among the several novel custom power devices, the STATCOM is the most technically advanced and economical device for voltage sag mitigation in distribution systems. The conventional STATCOM functions by injecting AC voltages in series with the incoming three phase network, the purpose of which is to improve the voltage quality by an adjustment in the voltage magnitude, wave shape and phase shift. The voltage sag compensation involves the injection of real and reactive power to the distribution system. The reactive power requirement can be generated electronically within inverter of the STATCOM.

The most common choice for the control of the STATCOM is PI controller since it has a simple structure and it can offer relatively a satisfactory performance over a wide range of operation. The main problem of this simple controller is the correct choice of the PI gains and the fact that by using fixed gains, the controller may not provide the required control performance, when there are variations in the system parameters and operating conditions. Therefore, online tuning process must be performed to insure that the controller can deal with all the variations in the system.[1]

Fuzzy logic, the logic of approximate reasoning, continues to grow in importance, as it provides an inexpensive solution for controlling ill-known complex systems. Fuzzy controllers have received adequate attention in motion control systems as they posses non-linear characteristics and a precise model is most often unknown. Fuzzy controller is already applied to STATCOM [5,7]. 
In many sensitive loads such as medical equipment and adjustable speed motor drives, this level of sensitivity particularly THD index may be very important. In few researches, improving voltage THD index has also been considered as an objective and a control criterion as well. However, in the aforementioned studies, algorithms with high level of complexity have been used meanwhile they may cause some problems in practical implementation. Also, we can claim that $[6,20]$ is the only research which has considered THD voltage as a second goal. In order to have an appropriate performance during voltage flash and to be able to decrease the sensitive load voltage THD, a two-objective optimization has been proposed in this paper. Hence, in this method, voltage sag will be the first objective and voltage THD will be considered as the second objective in STATCOM control system.

The tuning of fuzzy controller is a heuristic work. To eliminate such problems the evolutionary

techniques have been applied in solving the tuning of FLC parameters. The Genetic Algorithm, Ant Colony Optimization and GA which are the forms of probabilistic heuristic algorithm that have been successfully used in optimization of Fuzzy logic controller. The GA method is usually faster because the GA has parallel search techniques which emulate natural genetics operations.

The GA (GA) has been applied to optimization of FLC. In recent years GA has gained much popularity in different kinds of applications because of its simplicity, easy implementation and reliable convergence [17,22]. It has been found to be robust in solving continuous non-linear optimization problems. Both voltage sag and THD can be modified by the aforesaid algorithm.

On the other hand, to investigate the efficiency of the proposed algorithm; performance of STATCOM compensator during various faults in a typical network has been tested and compared with some controllers that were introduced before. STATCOM operation, multi-objective optimization with fuzzy membership function, and GA algorithm have been introduced and discussed in the following sections. After that, we have introduced the proposed method of the paper, and the final section contains the simulation results.

\section{BASIC CONCEPTS OF STATCOM}

A STATCOM is a solid state power electronics switching device consisting of either MOSFET or IGBT, a capacitor bank as an energy storage device and injection transformers. It is linked in shunt between a distribution system and a load that shown in Figure.1. The basic idea of the STATCOM is to inject a controlled voltage generated by a forced commuted converter in a parallel to the bus voltage by means of an injecting transformer. At normal operating condition, the STATCOM injects only a small voltage to compensate for the voltage drop of the injection transformer and device losses. However, when voltage sag occurs in the distribution system, the STATCOM control system calculates and synthesizes the voltage required to preserve output voltage to the load by injecting a controlled voltage with a certain magnitude and phase angle into the distribution system to the critical load.

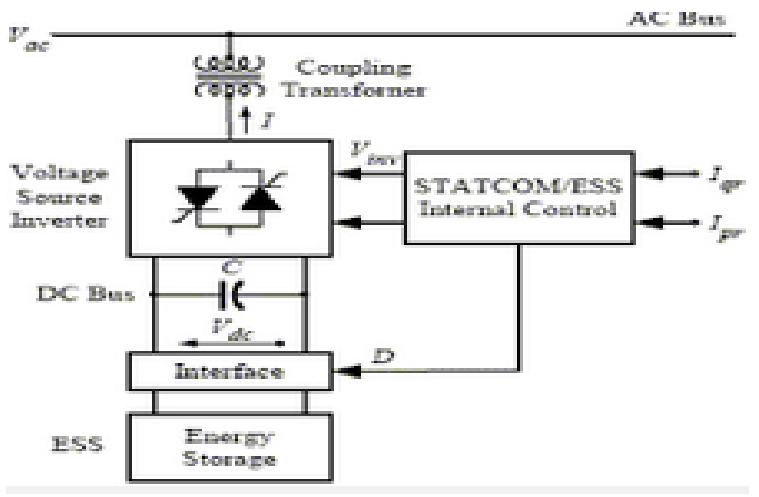

Figure 1. Proposed STATCOM Control Implementation

\section{GA ALGORITHM}

Genetic algorithms [9]-[10], which are adopted from the principle of biological evolution, are efficient search techniques that manipulate the coding representing a parameter set to reach a near optimal solution. Hence by strengthening fuzzy logic controllers with genetic algorithms the searching and attainment of optimal fuzzy logic rules and high-performance membership functions will be easier and faster. GAs is 
used regularly to solve difficult search, optimization and machine-learning problems that have previously resisted automated solutions. They can be used to solve difficult problems quickly and reliably. These algorithms are easy to interface with existing simulations and models, and they are easy to hybridize. GAs includes three major operators: selection, crossover, and mutation, in addition to four control parameters: population size, selection crossover and mutation rate. This paper is concerned primarily with the selection and mutation operators. There are three main stages of a genetic algorithm; these are known as reproduction, crossover and mutation.

The steps involved in genetic algorithm are described below.

- Start: Generate random population of $\mathrm{n}$ chromosomes (suitable solutions for the problem).

- Fitness: Evaluate the fitness $\mathrm{f}(\mathrm{x})$ of each chromosome $\mathrm{x}$ in the population.

- New population: Create a new population by repeating following steps until the new population is complete.

- Selection: Select two parent chromosomes from a population according to their fitness (the better fitness, the bigger chance to be selected).

- Crossover: With a crossover probability, cross over the parents to form new offspring (children). If no crossover was performed, offspring is the exact copy of parents.

- Mutation: With a mutation probability, mutate new offspring at each locus (position in chromosome).

Accepting: Place new offspring in the new population.

- Replace: Use new generated population for a further run of the algorithm

- Test: If the end condition is satisfied, stop, and return the best solution in current population.

- Loop: Go to step 2

\section{OBJECTIVE FUNCTIONS}

The main objective of controller is as follows

Minimization of Average Voltage Deviation The voltage deviation index is defined as the deviation of the voltage magnitude of bus i from the unity as where Vi-ref and Vi are the reference and actual voltages at

$$
\mathrm{V}_{\mathrm{dev}-\mathrm{i}}=\left(\mathrm{V}_{\mathrm{i}-\mathrm{ref}}-\mathrm{V}_{\mathrm{i}}\right)^{2}
$$

Bus $i$, respectively. Therefore, the average voltage deviation in the system per unit (p.u.) can be expressed using the summation of normalized $\mathrm{V}_{d e v-i} i$ for all buses given by

$$
f_{i}=V_{\text {dev-avr }}=\frac{\sum_{i=1}^{m} V_{d e v-i}^{\text {norm }}}{M}
$$

where $M$ is the total number of system buses.

Minimization of Average Voltage Total Harmonic Distortion (THDV) :The average of the normalized THDV in the system buses to control the THDV level of the whole system is obtained using

$$
\mathrm{f}_{2}=\mathrm{THD}_{\mathrm{v}-\mathrm{avr}}=\frac{\sum_{\mathrm{i}=1}^{\mathrm{m}} \mathrm{THD}_{\mathrm{v}-\mathrm{i}}^{\mathrm{norm}}}{\mathrm{M}}
$$

Where ${ }^{T H D_{v-i}^{n o r m}}$ I is the normalized $\mathrm{THD}_{\mathrm{v}}$ in bus $\mathrm{i}$.

Bus Voltage Limits: Each bus voltage $V i$ must be maintained around a permissible voltage band owing to the effect of D-STATCOM installation on system bus voltages. This is achieved using

$$
\mathrm{V}_{\mathrm{i}-\min } \leq \mathrm{V}_{\mathrm{i}} \leq \mathrm{V}_{\mathrm{i}-\max }
$$


where $V i$ is the voltage at bus $i$.

The overall optimal STATCOM problem can be configured as a constrained multi-objective optimization problem. Therefore, the weighted sum method is considered in the current study to combine the individual objective functions in terms of a single objective function. Each constraint violation is also incorporated in the overall objective function using the penalty function approach. The final objective function to be minimized is expressed as

$$
\mathrm{F}=\mathrm{w}_{1} \mathrm{f}_{1}+\mathrm{w}_{2} \mathrm{f}_{2}+\lambda \sum_{\mathrm{i} \in \mathrm{m}}\left[\max \left(\mathrm{V}_{\mathrm{i}}-\mathrm{V}_{\mathrm{i}-\max }, 0\right)+\max \left(\mathrm{V}_{\mathrm{i}-\max }-\mathrm{V}_{\mathrm{i}}, 0\right)\right.
$$

Where wi and $\lambda$ are the relative fixed weight factors assigned to the individual objectives and the penalty multipliers for violated constraints, respectively, and are large, fixed scalar numbers. In addition, $P$ and $M$ are the total STATCOM number and the total bus number, respectively.

The weight factors should be assigned to the individual objective functions based on their importance. These may vary based on the desired preferences of the power system operators. In this paper, the proper weighting factors used are $\mathrm{w} 1=\mathrm{w} 2=0.4$ in which the first two objectives are assumed to be equally more important.

\section{VOLTAGE SAG DETECTION}

The essential part for well-performance of controller in STATCOM is the sag detection circuit. Voltage sag must be detected fast and corrected with a minimum of false operations. The voltage sag detection method is based on Root Means Square (RMS) of the error vector which allows detection of symmetrical and asymmetrical sags, as well as the associated phase jump. The controller system is presented in Figure 2. The three-phase supply voltage is transformed from abc to odq frame using Park transformation. Phase Locked Loop (PLL) is used to track supply voltage phase.

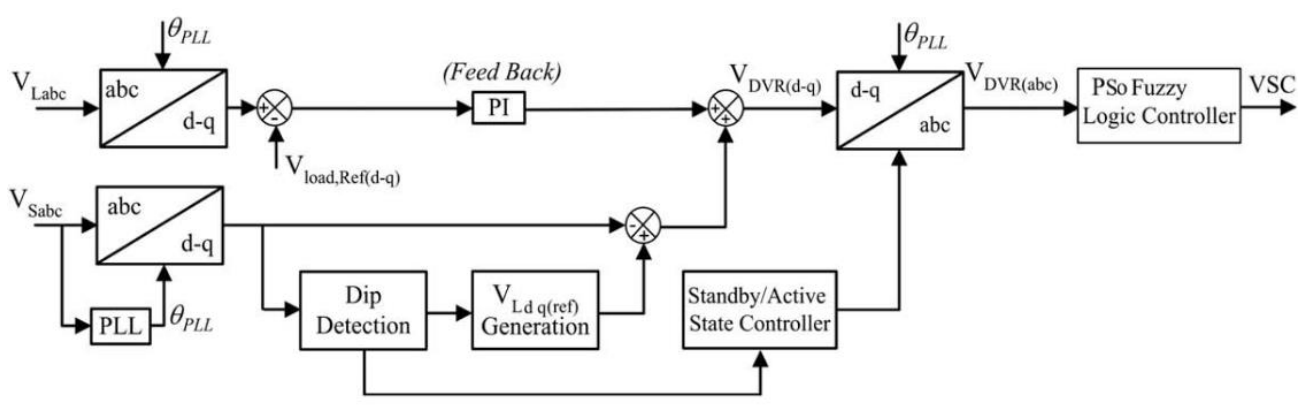

Figure 2. Control Structure of DVR

The park transformation matrix is shown as follows:

$$
\begin{aligned}
& V_{o}=\frac{1}{3}\left(V_{a}+V_{b}+V_{c}\right) \\
& V_{d}=\sqrt{\frac{2}{3}}\left[V_{a} \cos (\theta)+V_{b} \cos \left(\theta-\frac{2 \pi}{3}\right)+V_{c} \cos \left(\theta-\frac{4 \pi}{3}\right)\right] \\
& V_{q}=\sqrt{\frac{2}{3}}\left[V_{a} \sin (\theta)+V_{b} \sin \left(\theta-\frac{2 \pi}{3}\right)+V_{c} \sin \left(\theta-\frac{4 \pi}{3}\right)\right]
\end{aligned}
$$




$$
\left.\left|V_{s}\right|=\sqrt{V_{d}^{2}+V_{q}^{2}}\right)
$$

Closed loop load voltage feedback is added, and is implemented in the odq frame in order to minimize any steady-state error in the fundamental component. When the grid voltage is normal, the STATCOM system is held in a null state to lower its losses. When voltage sag is detected, the STATCOM switches into active mode to react as fast as possible to inject the required ac voltage. The injection voltage is also generated according to the difference between the reference load voltage and the supply voltage and it is applied to the converter to produce the preferred voltage, using the Voltage Control based on PSO-fuzzy logic controller.

By notice to standard GA algorithm, it can be find out the effect of population number over this algorithm. On the other word, low numbers of population is caused to stick in local optimum and if high numbers of population is caused to decline algorithm velocity. Therefore standard GA algorithm isn't profit for solving multi objective optimization problems.

In this paper, parameters of fuzzy logic controllers used for voltage sag and voltage THD are determined by GA. These parameters are input and output scaling factors, input membership function parameters and coefficients of linear output functions. GA iterations are performed by the MATLAB commands in an M-File. The MATLAB commands in M-File are as shown in Figure 3.

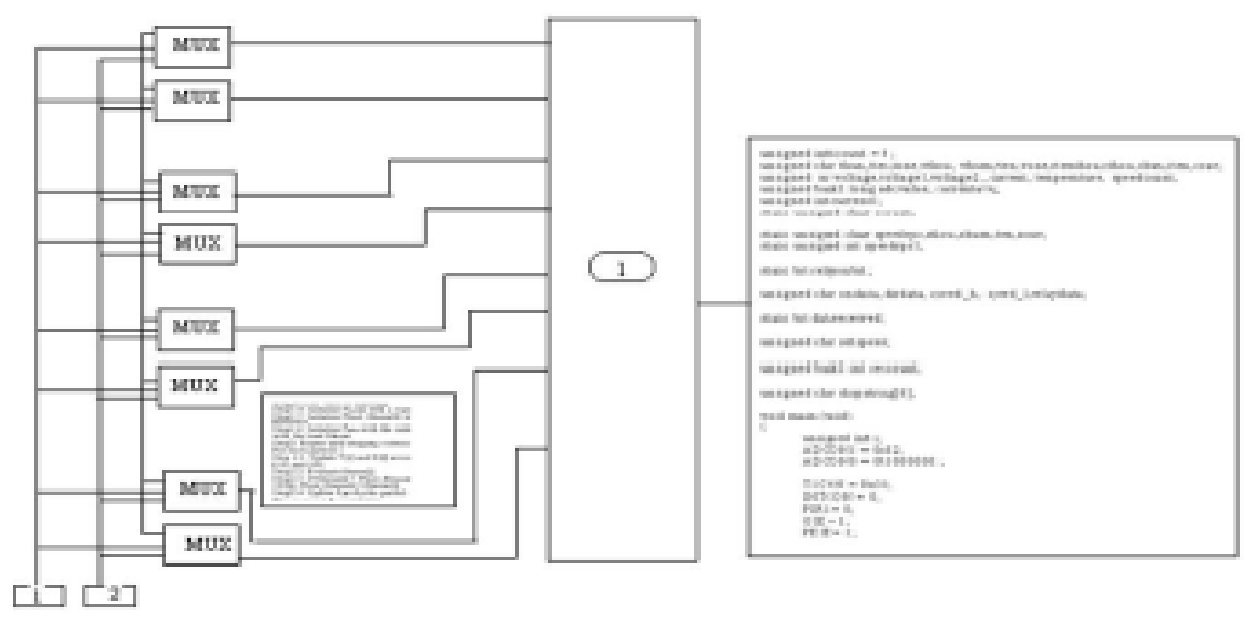

Figure 3. MATLAB Commands in M-File

The tuning process of FLC rules by GA is illustrated by the following steps.

1. Divide the input and output spaces of the system to be controlled into fuzzy regions.

2. Encode the input-output regions into bit-strings.

3. Use GA as a learning procedure to generate a set of fuzzy rules.

4. Use the newly generated fuzzy rules to determine the performance and assign a fitness value.

5. Assign a negative value to the fitness function if there any problem persists.

6. If stopping criterion is not met go to Step 3.

7. Determine a mapping from the input space to the output space based on the combined fuzzy rule base using a defuzzifying procedure. This paper presents a quick solution to the problems using the GA algorithm.

\section{RESULTS AND DISCUSSIONS}

The proposed algorithm is evaluated for STATCOM system to enhance the power quality issue. The power distribution system case study consists of two load buses that one of them includes the sensitive load. This simple electrical network has been shown in Figure 4 and its parameters have been introduced in Table 1.This project has been simulated in the MATLAB/SIMULINK. 


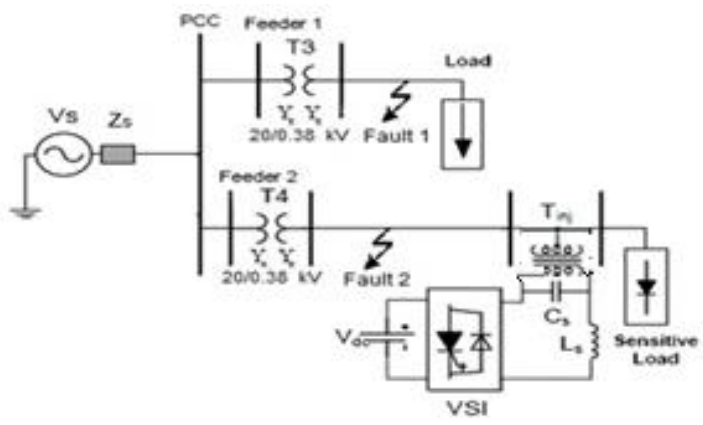

Figure 4. Power Distribution System Schematic

Table 1. Network Parameter

\begin{tabular}{ll}
\hline \multicolumn{1}{c}{ Parameters } & \multicolumn{1}{c}{ Values } \\
\hline Network Frequency Power & $\mathrm{Fn}=50(\mathrm{~Hz})$ \\
Supply Voltage & $\mathrm{Vs}=22500(\mathrm{~V})$ \\
Active And Reactive Power For & $\mathrm{P}=2000(\mathrm{~W})$ \\
Sensitive Load & $\mathrm{Q}_{1}=40(\mathrm{Var})$ \\
& $\mathrm{Qc}=10(\mathrm{Var})$ \\
Active And Reactive Power For & $\mathrm{P}=2500(\mathrm{~W})$ \\
Non-Sensitive Load & $\mathrm{Q}=40(\mathrm{Var})$ \\
Distribution Transformer Rated & $\mathrm{Pn}=3200(\mathrm{~W})$ \\
Power And Ratio & $20000 / 380$ \\
Distribution & $\mathrm{Rl}=0.0003(\mathrm{P} . \mathrm{U})$. \\
Impedances & $\mathrm{Xl}=0.001(\mathrm{P} . \mathrm{U})$. \\
& $\mathrm{Rm}=\mathrm{Xm}=500(\mathrm{P} . \mathrm{U})$. \\
Series Transformer Rated Power & $\mathrm{Pn}=1500(\mathrm{~W})$ \\
And Ratio & $100 / 1000$ \\
Series Transformer Rated Power & $\mathrm{Rl}=0.00001(\mathrm{P} . \mathrm{U})$. \\
And Ratio Impedances & $\mathrm{Xl}=0.0003(\mathrm{P} . \mathrm{U})$. \\
& $\mathrm{Rm}=\mathrm{Xm}=500(\mathrm{P} . \mathrm{U})$. \\
Switching Frequency & $\mathrm{Fs}=10000(\mathrm{~Hz})$ \\
Series Filter Impedances & $\mathrm{Rs}=0.2(\Omega)$ \\
& $\mathrm{Ls}=6(\mathrm{MH})$ \\
\hline
\end{tabular}

The performance of the proposed Fuzzy GA (FGA) based optimization is evaluated by comparing its results with conventional Fuzzy logic controller and Genetic fuzzy controller. Algorithm begins with generation of initial population that contains 50 chromosomes. Each chromosome of this population is in the form of an array which consists of 22 columns. Each column indicates possible optimal value of corresponding FLC parameter

In this study, total error is used while selecting best individuals. For this purpose, in the M-file, generated parameters are loaded to the fuzzy logic controller by the command "set_param()" and STATCOM circuit is run in a Simulink Mdl-file by the command "sim()". During simulation, error is calculated and total error is sent to an array. This procedure is performed for each chromosome of initial population and finally an array with 50 rows is formed.

After the production of initial population and calculating total error for each chromosome in the Population, GA iterations begin. Finally, STATCOM system is made to run again using new parameters to obtain new fitness values. This cycle terminates when predetermined number of iteration is reached. The fittest chromosome of each population is stored in an array and at the end of the iterations the fittest chromosome of all populations is obtained.

Table 2 represents the parameter chosen for implementation of GA-FLC and table 3 represents the parameter chosen for implementation of FGA.

Table 2 Parameters Chosen for GA Implementation

\begin{tabular}{cc}
\hline Parameter for GA-FLC & Value \\
\hline Population size & 20 \\
No. of iteration & 100 \\
Crossover probability & 0.9 \\
Mutation probability & 0.1 \\
Selection & Roulette wheel \\
\hline
\end{tabular}


The simulated structure of optimized fuzzy controller for the STATCOM system is shown in figure 5. Number of dimensions in the problem is four. These dimensions are the coefficients of two PI controllers so that one of them is aligned with the d-axis and the other is aligned with the q-axis. Each PI controller has two coefficients, i.e, proportional and integral gains.

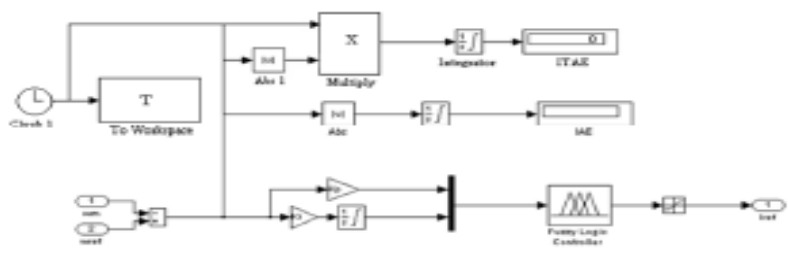

Figure 5. Simulated Structure of Optimized Fuzzy Controller

For both blocks (error signal-d and q) the FL controller consists of three linguistic variables from input which is; Negative $(\mathrm{N})$, Zero $(\mathrm{Z})$ and Positive $(\mathrm{P})$. Each parameter from linguistic variables for error signal. Figure 6,7 and 8 shows the rule viewer and surface viewer of the fuzzy controller employed respectively.

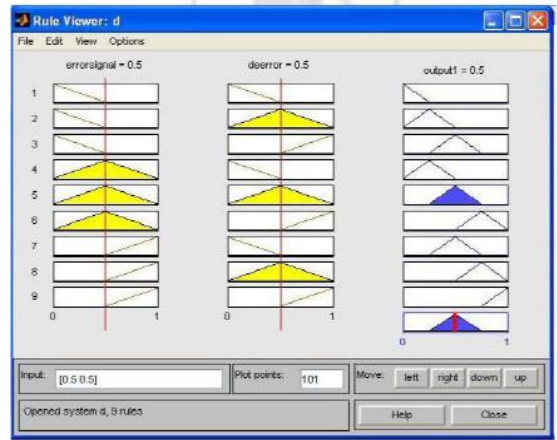

Figure 6. Rule Viewer of d Ref

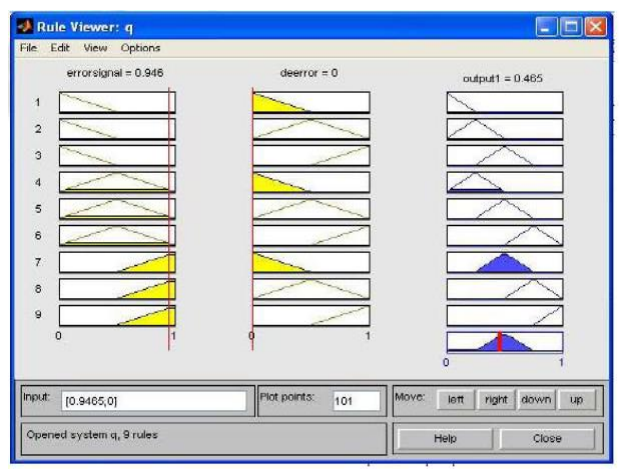

Figure 7. Rule Viewer of q Ref

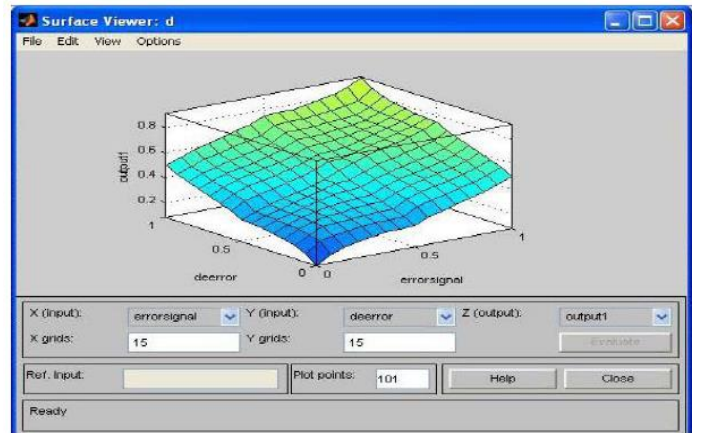

Figure 8. Surface Viewer

In order to simulate more critical conditions, two faults have been simulated. The first fault is just after series injection transformer with fault and earth resistances equal to $4.6 \Omega$ and $0.1 \Omega$ respectively, and the second one is near to a non sensitive load with the same resistance values. Simulation results revealed.

An improvement in the voltage sag and voltage THD of sensitive load. Figure 9 (a) and (b) shows voltage signals at PCC, the sensitive load voltage, injected voltage from STATCOM and the sensitive load voltage deviation signal from the base voltage in GA and GA-FLC case under fault conditions respectively. 


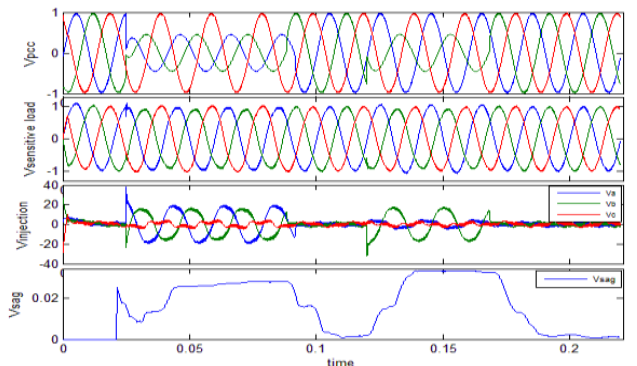

(a)

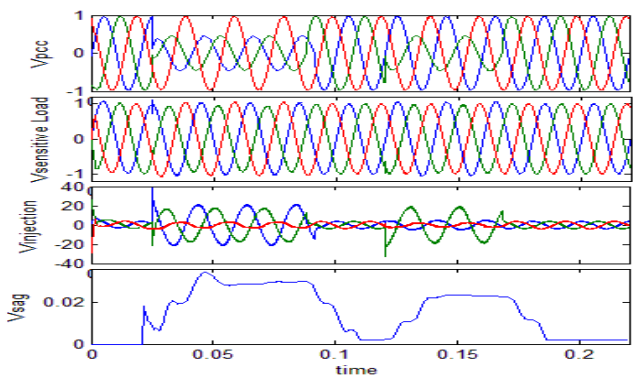

(b)

Figure 9. (a) and (b) Shows Voltage Signals At PCC, The Sensitive Load Voltage, Injected Voltage from STATCOM and the Sensitive Load Voltage Deviation Signal from the Base Voltage in GA and GA-Fuzzy Case Under Fault Conditions Respectively

The STATCOM is able to produce the required voltage components for different phases rapidly and help to maintain a balanced and constant load voltage at $1.00 \mathrm{pu}$. We can notice that during normal operation, the STATCOM is doing nothing but once voltage sag is detected, it quickly injects necessary voltage components to smooth the load voltage.

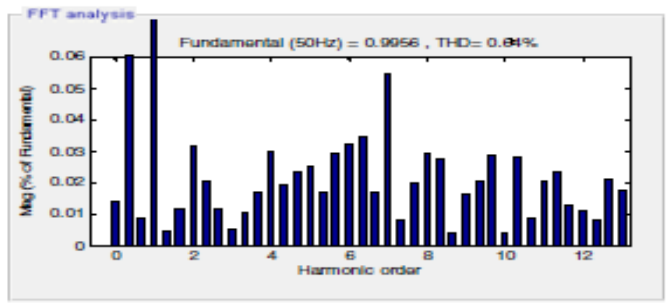

Figure 10. FFT Analysis for GA-FC

Figure 10 shows the Total Harmonic Distortion (THD) for GA-FC is $0.84 \%$. Improvement in THD signal and also deviation signal in line voltage caused by network faults in the algorithm, have been studied. As it can be seen, during simulation the requirements of IEEE-519 standard have been considered. The result has been shown in Table 3.

Table 3 Comparison of Results

\begin{tabular}{cccccc}
\hline Controller & Fault location & \multicolumn{2}{c}{ Voltage sag average } & \multicolumn{2}{c}{ THD (\%) } \\
& & value & $\begin{array}{c}\text { Improvement } \\
(\%)\end{array}$ & value & $\begin{array}{c}\text { Improve } \\
(\%)\end{array}$ \\
\hline Classical PI & 1 & 0.0205 & - & 4.87 & - \\
& 2 & 0.0273 & - & 3.94 & - \\
Conventional & 1 & 0.0177 & 13.66 & 1.46 & 70.02 \\
GA & 2 & 0.0203 & 25.64 & 1.59 & 59.64 \\
GA-FLC & 1 & 0.0131 & 36.09 & 0.84 & 82.75 \\
& 2 & 0.0178 & 34.79 & 0.91 & 76.90 \\
\hline
\end{tabular}

\section{CONCLUSION}

New ideas presented in this paper can be analyzed. In this paper, it is tried to achieve a better set of coefficients for PI controller using GA-Fuzzy algorithm. As the results show, the PI adjusted by this algorithm is better than classic PI controller. Adjusting controller coefficients using a GA-FLC optimization algorithm in order to improve voltage THD and voltage sag indices. In this paper a new bi-objective optimization algorithm is used for adjusting PI coefficients. This bi-objective algorithm is based on the 
fuzzification of aforementioned objectives. Simulation results revealed better performance of this GA-FLC Algorithm over the genetic algorithm from voltage sag and voltage THD.

\section{REFERENCES}

[1] P.K.Nandan and P.C.Sen (1986). A Comparative Study of PI and IP Controllers for DC Motor Drives. International Journal of control, vol.44,pp-283-297

[2] L. Ljung (1987) System Identification: Theory for the User. New York: Prentice-Hall.

[3] K.Kristinsson and G.A. Dumont (1992) System identification and control using genetic algorithm. IEEE Trans.Syst., Man, Cyber, vol.22, No.5, pp.1033-1046.

[4] J. Kennedy, R. Eberhart , "GA", Proc. IEEE International Conf. on Neural Networks (Perth, Australia), IEEE Service Center, Piscataway, NJ, pp. 1942-1948, 1995

[5] A.Visoli (1999), Fuzzy logic based Set-point weight tuning of PID controllers. IEEE Transactions on systems, man and cybernetics- part A: Systems and Humans, vol.29, No.6.

[6] A.Besharati, W.Lo and K.M.Tsang, (1997) Self -tuning of PID controller using Newton-Raphson search method. IEEE Trans. On Industrial Electronics, vol.44, No.5, pp.717-725

[7] B. Wu and X. Yu (2000) Fuzzy modeling and identification with genetic algorithm based learning. Fuzzy Sets Syst., vol. 113, no. 3, pp. 351-365,Aug

[8] A.Visioli (2001), Tuning of PID controllers with fuzzy logic. IEEE proceeding-Control theory and Applications, vol.148, 1, pp1-8.

[9] R.A.Krohling and J.P.Rey (2001) Design of optimal disturbance rejection PID controller using genetic algorithm. IEEE Trans. Evol. Comp, vol.5, pp.78-82, Feb.

[10] K. M. Chow and A. B. Rad (2002) On-line fuzzy identification using genetic algorithms. Fuzzy Sets Syst., vol. 132, no. 2, pp. 147-171, Dec.

[11] R. Caponetto, L. Fortuna, S. Fazzino, and M. G. Xibilia, "Chaotic sequences to improve the performance of evolutionary algorithms," IEEE Trans. on Evolutionary Computation, Vol. 7, No. 3, pp. 289-304,Jun. 2003

[12] Zhang Zhihao and Wu Jianwen(2014), "Negative sequence current feed-forward control for three-phase four-leg STATCOM under unbalanced loads" International Conference on Power System Technology,Page(s): 2202 - 2208

[13] Al-Ismail, F.S. ; Hassan, M.A. ; Abido, M.A (2014). "RTDS Implementation of STATCOM-Based Power System Stabilizers", Canadian journal of electrical and Computer Engineering, Volume: 37 , Issue: 1 , , Pg:48 - 56

[14] Varma, R.K. ; Rahman, S. ; Vanderheide, T.(2014) "New Control of PV Solar Farm as STATCOM (PV-STATCOM) for Increasing Grid Power Transmission Limits During Night and Day, Volume:6, PP.99 